\title{
RESEARCH
}

\section{The dual role of RFX6 in directing $\beta$ cell development and insulin production}

\author{
Jing Lu1', Cheng Cheng1', Zhen-Chao Cheng2,3, Qian Wu²,4, Han Shen'1, Ming-xia Yuan', Bo Zhang² and Jin-Kui Yang1 \\ 1Beijing Key Laboratory of Diabetes Research and Care, Beijing Diabetes Institute, Beijing Tongren Hospital, Capital Medical University, Beijing, China \\ ${ }^{2}$ Key Laboratory of Cell Proliferation and Differentiation of the Ministry of Education, College of Life Sciences, Peking University, Beijing, China \\ ${ }^{3}$ Beijing School, Beijing, China \\ ${ }^{4}$ HangZhou XueJun High School, Hangzhou, China
}

Correspondence should be addressed to J-K Yang: jkyang@ccmu.edu.cn

\begin{abstract}
RFX6 transcription factor is believed to play a central role in directing cell development of insulin-producing pancreatic islet. RFX6 homozygous mutations cause syndromic neonatal diabetes with hypoplastic pancreas. However, RFX6 heterozygous mutations cause maturityonset diabetes of the young (MODY) with normal pancreas development. Here, we show that RFX6 may control islet cell development and insulin production in different manners. The rfx6 knockout zebrafish generated by CRISPR/Cas9 exhibited an overt diabetes phenotype. Pancreatic islet failed to form compact structures in the knockout fish. While endocrine pancreatic islet non- $\beta$-cells were absent, insulin-producing $\beta$-cells were present in the knockout fish. Although insulin mRNA level was normal in the $\beta$-cells of the knockout fish, insulin protein level was decreased. High-throughput RNA sequencing (RNAseq) showed that differentially expressed genes were enriched in the translation term in islet $\beta$-cells from the knockout fish. Chromatin immunoprecipitation sequencing (ChIPseq) of normally developed islet $\beta$-cells from mice demonstrated that $r f \times 6$ interacted with translation initiation factors and controlled insulin translation. Our data indicate that Rfx6 may act as a transcription factor regulating the transcription of genes involved in mRNA translation, which may represent a new mechanism and treatment strategy for diseases.
\end{abstract}

\section{Key Words}

- Rfx6

- islet development

$\checkmark$ insulin

- RNA-seq

- ChIP-seq

\author{
Journal of Molecular \\ Endocrinology \\ (2021) 66, 129-139
}

\section{Introduction}

Diabetes is a global health problem that is tightly associated with the dysfunction or loss of insulinproducing pancreatic islet $\beta$-cells. In this context, seeking the mechanisms underlying $\beta$-cell development and function serves a crucial challenge. Specific transcription factors including PDX1, NEUROD, NKX2.2, NKX6.1 and PAX6 are involved in the development of pancreatic islet $\beta$-cells (Desai et al. 2008, Papizan et al. 2011, Taylor et al. 2013, Pauerstein et al. 2015, Zhu et al. 2016).

The regulatory factor X6 (RFX6) transcription factor is expressed at a high level only in the pancreas (Smith et al.
2010). Homozygous mutations in RFX6 cause MitchellRiley syndrome, an autosomal recessive syndrome of severe neonatal diabetes, hypoplastic or annular pancreas and intestinal atresia (Smith et al. 2010, Pearl et al. 2011, Concepcion et al. 2014). It has been proposed that this severe diabetes in patients with homozygous mutations in RFX6 contributed to the overall deficiency of pancreatic islet development and function including $\beta$-cell insulin production (Smith et al. 2010, Pearl et al. 2011). On the other hand, heterozygous mutations in RFX6 can cause mild maturity-onset diabetes of the young (MODY) with 
reduced penetrance (Patel et al. 2017). In these patients with RFX6-MODY, defective insulin secretion was attributed to insulin-secretive function failure of the islet $\beta$-cells with normal development of the islets (Chandra et al. 2014, Piccand et al. 2014). Therefore, we propose that RFX6 controls both pancreatic islet development and insulin production in different manners.

RFX6 knockdown in human EndoC-BH1 cells showed changes in insulin mRNA (Chandra et al. 2014). Mice with $R f \times 6$ knockout die within 2 days after birth, and hence the role of RFX6 in $\beta$-cell function and insulin production is limited (Smith et al. 2010, Pearl et al. 2011). In this study, we used $r f x 6$ knockout zebrafish (Danio rerio) generated by CRISPR/Cas9 to illustrate the precise role of $r f x 6$ in controlling insulin production in fetal and postnatal stages (Zhang et al. 2016b). To study the role of $r f x 6$ in controlling insulin production in postnatal stages in animals with normal development, we also performed chromatin immunoprecipitation sequencing (ChIPseq) in mice. Our study shows that $r f x 6$ is both a transcription factor essential for non- $\beta$-cell development and a translation factor or initiator for insulin protein translation.

\section{Materials and methods}

\section{Zebrafish strains and maintenance}

The endocrine pancreas was isolated from the bacterial artificial chromosome neurod1:GFP transgenic fish (Obholzer et al. 2008) and insulin:mCherry transgenic fish (Pisharath et al. 2007). Adult neurod1:GFP, neurod:GFP/ins ulin:mCherry and WT (Tubingen strain) zebrafish (Danio rerio) were raised and cared for according to standard protocols in a recirculating system (The Aquatics Habitat, Gloucester, UK). After treatment, the experimental fish were maintained in isolation tanks with daily water changes. All experiments with laboratory animals were complied with the Ethical Review Committee at Beijing Tongren Hospital, Capital Medical University, China (No. TRECKY2018-037).

\section{CRISPR/Cas9 design}

The CRISPR/Cas9 gene sequence was designed and ordered commercially (Sangon, Shanghai, China). The full-length products were cloned into pcDNA3.1 (Invitrogen). The human U6 promoter and gRNA scaffold were obtained commercially (Sangon) and cloned into the pUC19 vector. The sequence of Cas9 was designed as 5'-GAAGAAGACCTGGGCCATCTGG-3'.

\section{Blood glucose determination}

Blood glucose measurements were obtained as follows: zebrafish were anesthetized in $4^{\circ} \mathrm{C}$ ice-water for $2 \mathrm{~min}$, and a pulled capillary pipet was inserted into the atrium of the beating heart. Approximately $5 \mu \mathrm{L}$ of blood was removed and rapidly transferred to a Freestyle Freedom Lite device. We did not use blood from the tail vein because this technique caused more lasting damage than heart puncture. Using the Freestyle Freedom Lite device, $0.3 \mu \mathrm{L}$ of blood was sufficient for glucose measurement.

\section{Immunohistochemistry analysis of zebrafish tissue}

Tissues were fixed by immersion in 4\% paraformaldehyde for $4 \mathrm{~h}$ at room temperature, then embedded in paraffin. Heat-induced antigen retrieval was then performed on the paraffin-embedded sections $(5 \mu \mathrm{m})$ in citrate buffer $(\mathrm{pH}$ 6.0) at $99^{\circ} \mathrm{C}$ for $5 \mathrm{~min}$ by using a microwave. The slides were incubated in normal serum at room temperature for $1 \mathrm{~h}$, followed by incubation with the primary antibody, guinea pig anti-insulin antibody; diluted 1:100 (Abcam) or rfx6 antibody PA5-68655; diluted 1:100 (Thermo). The sections were washed three times with PBS and then incubated with Alexa Fluor 555 guinea pig anti-goat; diluted 1:200 (SC-362265, Santa Cruz). These sections were mounted with fluorescent mounting medium containing DAPI (ZSGB-BIO, Beijing, China). Hematoxylin and eosin (H\&E) staining was used to observe islet morphology changes. Images were recorded with a laser-scanning confocal microscope, Leica TCSSP5 (Leica).

\section{Western blotting}

The pancreas were solubilized in radioimmunoprecipitation assay (RIPA) buffer containing 100 $\mathrm{mmol} / \mathrm{L}$ phenylmethylsulfonyl fluoride (PMSF) and 25 $\mathrm{mmol} / \mathrm{L}$ proteinase inhibitors (Roche). The supernatant was collected following centrifugation of the lysate at $10,000 \boldsymbol{g}$ for $20 \mathrm{~min}$ at $4^{\circ} \mathrm{C}$ and the enhanced BCA Protein Assay Kit (Beyotime Biotechnology, Beijing, China) was used to determine protein concentration in the samples. Equivalent amounts of protein were separated by $10 \%$ SDS-PAGE and transferred to polyvinylidene fluoride (PVDF) membranes (Millipore). The membrane was blocked with 5\% non-fat dry milk for $1 \mathrm{~h}$ followed by immunoblotting with specific antibodies (1:1000, ab210560, Abcam). Western blotting images were scanned and analyzed with ImageJ software for densitometry measurements. 


\section{RT-PCR and real-time RT-PCR}

Total RNA was purified using an RNA prepPure Tissue Kit (Tiangen Biotech, Beijing, China). RNA samples were converted to cDNA using FastQuant RT Super Mix (Tiangen Biotech). Real-time RT-PCR was then performed on a LightCycler® 480 Real-Time PCR System (Roche) using SYBR Green I Master Mix reagent (Roche). Three distinct amplifications were performed in duplicate for each transcript, and mean values were normalized to the mean value of the reference mRNA cyclophilin.

\section{RNA probes, whole-mount in situ hybridization (WISH) and imaging}

Antisense RNA probes were prepared by transcribing linearized cDNA clones with SP6, T7 or T3 polymerase using digoxigen in or DNP labeling mix (Roche) according to the manufacturer's instructions. The probes were subsequently purified on NucAway spin columns (Thermo) and ethanol-precipitated. The zebrafish insulin, glucagon, somatostatin, ghrelin and trypsin probes were used. Single whole-mount and double fluorescent in situ hybridizations were performed as described (Tong et al. 2014). For visible WISH, the numbers of cells were quantified by counting the cells under the microscope by focusing successively on each layer of stained cells.

\section{RNAseq}

RNAseq experiment was performed from isolated pancreas of zebrafish. A total of $3 \mu \mathrm{g}$ of RNA per sample was used as an input material for RNA sample preparation. Sequencing libraries were generated using the NEB Next ${ }^{\circledR}$ Ultra $^{\mathrm{TM}}$ RNA Library Prep Kit for Illumina ${ }^{\circledR}$ (New England Biolabs, MA, USA). The index-coded samples were clustered using a cBot Cluster Generation System and a TruSeq SR Cluster Kit v3-cBot-HS (Illumina) according to the manufacturer's instructions. After cluster generation, the library preparations were sequenced on an IlluminaHiSeq 2000/2500 platform, and 150-bp/100-bp/50-bp paired/ single-end reads were generated.

\section{Functional analysis of differentially expressed genes}

The gene ontology (GO) enrichment analysis and the webbased Kyoto Encyclopedia of Genes and Genomes (KEGG) pathway enrichment analysis was provided by DAVID Bioinformatics Resources 6.7 (http://david.abcc.ncifcrf. gov). Gene functional enrichment was performed using the default parameters in DAVID to obtain an adjusted $P$-value $<0.05$ for the test gene group vs the zebrafish COG ontology annotation set.

\section{ChIPseq}

For ChIP, tissue preparation and ChIP protocol closely adhered to steps outlined in the SimpleChIP ${ }^{\circledR}$ Plus Sonication Chromatin IP System manual \#56383 (Cell Signaling Technology), according to manufacturer's instructions as described previously (Cheng et al. 2019). Several antibodies were used to perform ChIP assay. We found that the most efficient antibody was from Millipore (\#ABD28). Chipseq experiment were repeated three times.

\section{ChIP-qPCR}

ChIP was performed using a protocol that described previously for ChIPseq. qPCR data were generated using a LightCycler96 thermocycler (Roche) and the qPCR SuperMix (Transgen Biotech, Beijing, China) according to manufacturer's instructions.

\section{Statistics and data analysis}

All statistical analyses were conducted with MedCalc version 11.4 (http://www.medcalc.be) or GraphPad Prism version 6.0. Data are presented as means \pm S.E.M., and statistical comparisons were performed using Student's $t$ tests or the Mann-Whitney $U$ test as appropriate. Significance was defined as $P<0.05$. In gene ontology and DAVID analysis, the statistical data were generated by the software.

\section{Results}

\section{Rfx6 knockout causes diabetes in zebrafish}

We designed a CRISPR/Cas9 short guide RNA (sgRNA) that specifically targets exon 5 of zebrafish $r f x 6$. Restriction site disruption assay and PCR sequencing were used to confirm the target site modification. Three different frameshift mutant lines were obtained, which we named $r f x 6^{+13}, r f x \sigma^{+14}$ and $r f x 6^{+22}$. We used both male and female fish in this study and found no genderspecific phenotypes. Western blotting results showed that the protein level of rfx6 decreased dramatically in KO fish which demonstrated that $r f \times 6$ mutant fish was successfully constructed (Fig. 1A). Each of the line were used to perform the next experiments. These three $r f x 6$ mutants showed decreased survival from approximately 
A
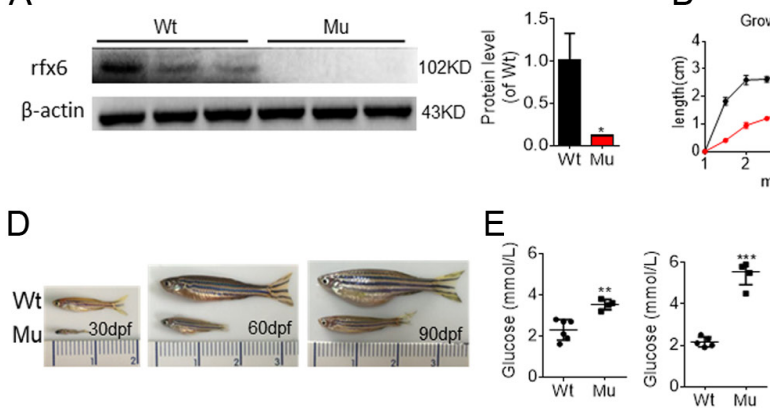

G

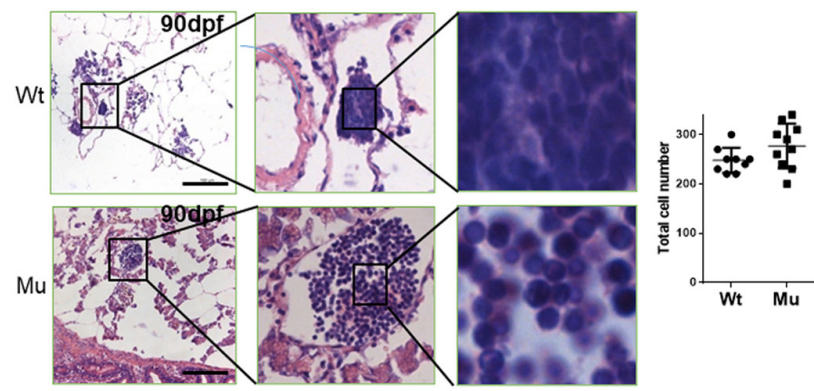

C

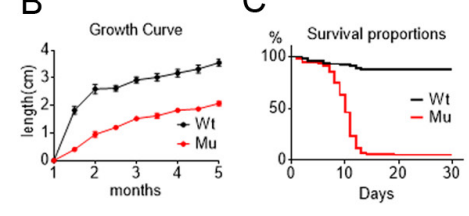

$\mathrm{F}$

\section{Figure 1}

Diabetes caused by mutation of $r f x 6$ in zebrafish (A) The protein level of $r f \times 6$ decreased dramatically in KO fish. (B, C, D and E) The $r f x 6$ knockout zebrafish model showed diabetic phenotype with smaller body shape at different stages (B), shorter growth curve (C), lower survival rates (D), elevated blood glucose at $60 \mathrm{dpf}$ (left) and $90 \mathrm{dpf}$ (right) (E) and impaired fin regeneration (E) compared with the WT zebrafish. (F) The pancreas of rfx 6 knockout fish were smaller than the WT at $90 \mathrm{dpf}(\mathrm{F})$. H\&E staining showed a loose, dispersed and larger shape in rfx6 knockout fish compared with adult WT (G, Scale Bars, $25 \mu \mathrm{m}) . n=10$ fish (A, B, C), $n=100$ fish (D), $n=6$ fish (E, F) and $n=8$ fish (G) per group for the mutant $(\mathrm{Mu})$ and $\mathrm{WT} . * * P<0.01, * \star * P<0.001$; Mann-Whitney $U$ test (G) Data are mean \pm S.E.M. $\mathrm{Mu}$, mutant. A full color version of this figure is available at https://doi.org/10.1530/JME-20-0119.
7 days post fertilization (dpf), with most fish dead by 14 dpf. Surviving mutants were infertile and exhibited a gaunt body type (Fig. $1 \mathrm{~B}$ and C). Only 5\% of mutant embryos survived to adulthood (Fig. 1D).

Previous reports have shown that mutations of human RFX6 resulted in neonatal diabetes (Smith et al. 2010, Pearl et al. 2011, Concepcion et al. 2014). When examining the glucose metabolism of $r f x 6$ knockout zebrafish, we found that, compared with their WT siblings, the adult rfx6 knockout fish exhibited a significantly increased blood glucose levels at both $60 \mathrm{dpf}$ and $90 \mathrm{dpf}$ (Fig. 1E), gaunt body type and diabetic complications including fin regeneration in zebrafish (Intine et al. 2013) (Fig. 1F). The $r f x 6$ knockout fish failed to cope with a cold stimulus, thus implying that $r f x 6$ may function in maintaining the stress response system of zebrafish. All of which is the characteristics of insulin-depending diabetes mellitus.

\section{$R f x 6$ is necessary for compact islet structure}

To further validate the morphological changes of islets form the adult $r f x 6$ knockout fish, we conducted hematoxylin and eosin (H\&E) staining histological experiments. According to reference, main pancreas of the adult zebrafish has one large islet and three to six smaller islets (Namba 2019, Toselli et al. 2019). The pancreas of the $r f \times 6$ knockout fish was smaller compared with WT. The WT islets presented compact and dense shapes, while the islets of $r f x 6$ knockout fish were significantly looser and larger. Total number of $\beta$-cell numbers and $\beta$-cell proliferation were unchanged both in $\mathrm{Wt}$ and $\mathrm{Mu}$ fish (Fig. 1G). These data show that $r f x 6$ is necessary for normal islet morphology.

\section{Rfx6 knockout decreases insulin protein production}

In this study, we choose a neurod1:eGFP transgene that is broadly expressed in the CNS during embryonic development as the target integration site (Obholzer et al. 2008). The $e G F P$ transgene allows the direct visualization of target gene disruption and should not compromise survival upon loss of gene function. We used neurod:eGFP line in this study. In WT embryos, neurod:eGFP transgenic fish showed eGFP expression within the compact islet structure at $3 \mathrm{dpf}$ (Fig. 2A). The numbers of neurod ${ }^{+}$cells were unchanged in rfx6 mutants, thus demonstrating that rfx6 mutation does not affect the early stages of pancreatic endocrine development (Fig. 2B). In WT islet cells, which were identified by neurod:eGFP, labeling of insulin showed clear insulin protein in the islets of embryo WT fish, while the knockout fish showed decreased insulin protein at the age of 3 dpf (Fig. 2B) and $90 \mathrm{dpf}$ (Fig. 2C).

We further examined whether insulin protein was influenced in the adult $r f \times 6$ knockout fish (90 dpf). Total number of $\beta$-cell numbers and $\beta$-cell proliferation were also unchanged both in $\mathrm{Wt}$ and $\mathrm{Mu}$ fish. Western and histochemical staining showed clear insulin protein in the islets of adult WT fish, while dramatically decreased insulin protein was detected in the islets of adult $r f \times 6$ knockout fish (Fig. 2D). 

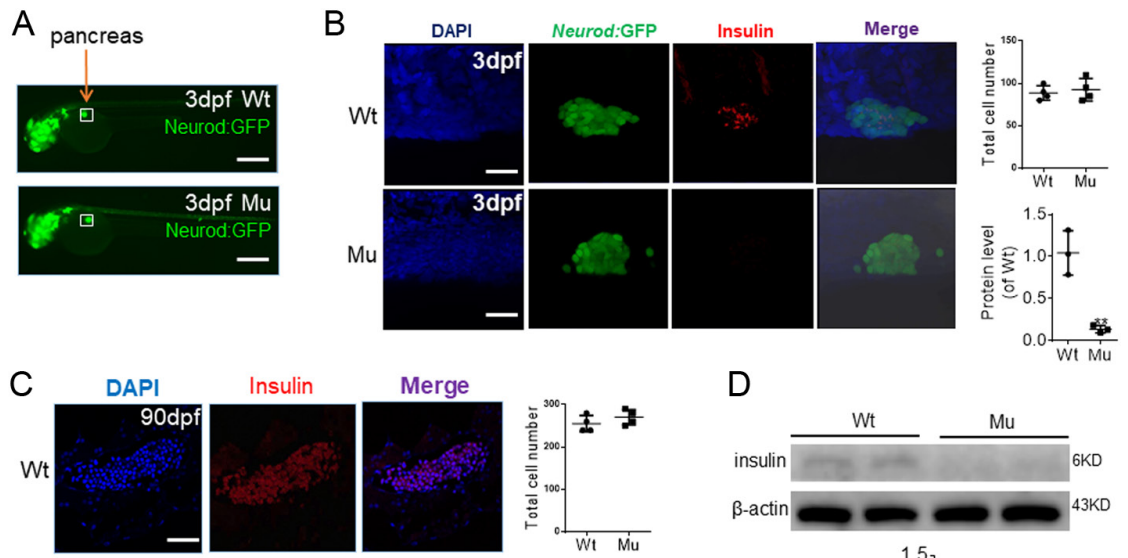
Merge
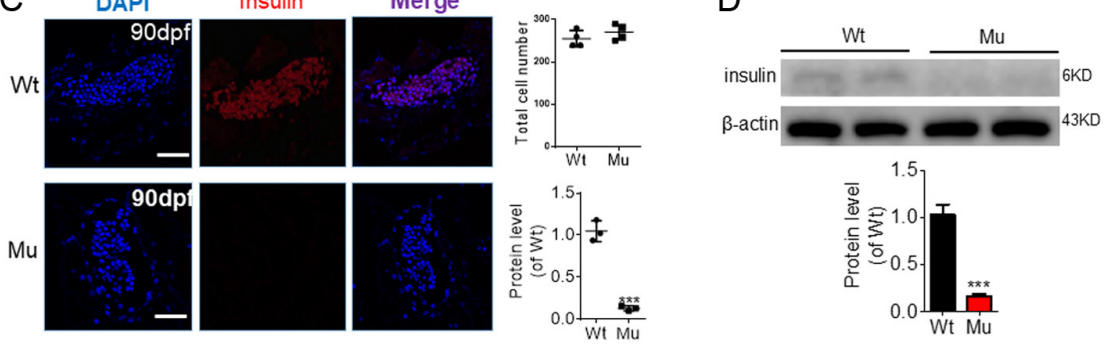

\section{Figure 2}

Significantly decreased insulin protein of $r f \times 6$ knockout fish. (A and B) Neurod:eGFP transgenic line was used to incross with $r f \times 6^{+/-}$line. $r f \times 6^{-/-/ n e u r o d: e G F P}$ showed green fluoresce (A, Scale Bars, $1 \mu \mathrm{m}$ ). (B and C) Significantly decreased insulin protein of $r f \times 6$ knockout fish were detected both in $r f \times 6^{-/-} /$neurod:eGFP in the embryos (B, Scale Bars, $100 \mu \mathrm{m}$ ) at the age of 3 dpf and $r f \times 6^{-/-}$adult fish (C, Scale Bars, $\left.10 \mu \mathrm{m}\right)$ at the age of $90 \mathrm{dpf} . n=4$ (B, C) independent samples per group for the mutant $(\mathrm{Mu})$ and WT. (D) Significantly decreased insulin protein were detected in Mu adult fish compared with Wt fish. $* * P<0.01, * * * P<0.001$; Mann-Whitney $U$ test (B, C, D). Data are mean \pm S.E.M. A full color version of this figure is available at https://doi.org/10.1530/ JME-20-0119.
These data show that the $r f \times 6$ mutation does not influence the development of $\beta$-cells and that the gene is necessary for the production of insulin protein and therefore for regulating glucose metabolism in postnatal stage.

\section{Rfx6 only regulates islet non- $\beta$-cells development}

Because $r f x 6$ was found to be necessary for the development of the endocrine pancreas, we next examined whether it affects exocrine pancreas development. At $4 \mathrm{dpf}, \quad r f \times 6$ knockout fish showed normal trypsin expression compared with that of WT animals, thus indicating that the development and function of acinar cells were normal (Fig. 3A). Briefly, $r f x 6$ did not influence the development of the exocrine pancreas, demonstrating that its effect is specific to the endocrine pancreas.

We next explored the islet cell development through whole-mount in situ hybridization (WISH) and RT-PCR. The results showed that mRNA levels of glucagon ( $g c g a)$, somatostatin (sst) and ghrelin (ghrl) were significantly downregulated in the $r f x 6$ knockout fish at $2 \mathrm{dpf}$, thus indicating a failure of $\alpha$-, $\delta$ - and $\varepsilon$-cell development (Fig. 3B). However, mRNA levels and sequence of insulin (ins) appeared normal in the $r f x 6$ knockout fish, suggesting that $\beta$-cell development remained unaffected (Fig. 3C). RNAseq results further confirmed that glucagon (gaga), somatostatin (sst) and ghrelin (ghrl) expression were downregulated in $r f \times 6$ knockout fish, and that insulin (ins) expression remained unaffected. The results showed that

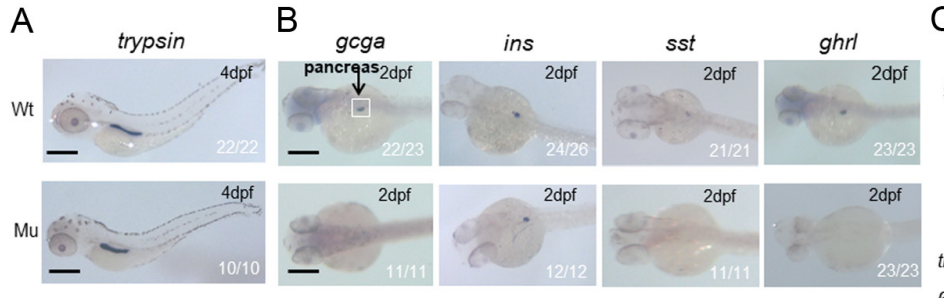

D

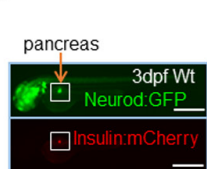

E

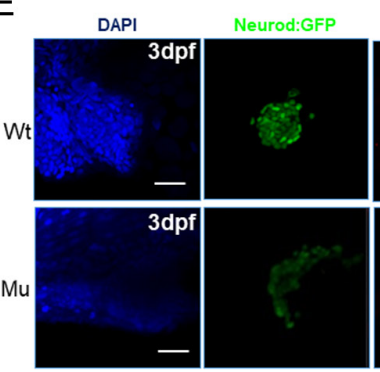

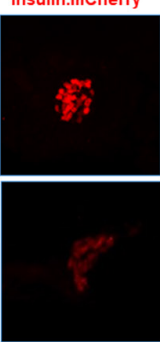

Merge

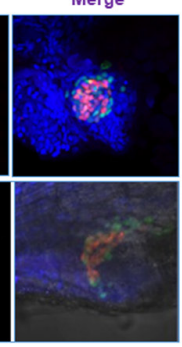

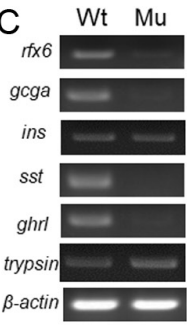

2dpf

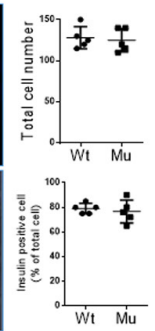

Figure 3

Failure of non- $\beta$ endocrine cell differentiation in the $r f \times 6$ knockout fish. (A and B) In situ hybridization showed that the expression levels of glucagon (gcga), somatostatin (sst) and ghrelin (ghrl) were decreased in the $r f \times 6$ knockout fish (B, Scale Bars, $20 \mu \mathrm{m}$ ), whereas trypsin (A, Scale Bars, 10 $\mu \mathrm{m}$ ) and insulin (ins) (B) level was normal. (C, D and E) Neurod:eGFP/insulin:mCherry transgenic line was used to incross with $r f \times 6^{+/-}$line (D, Scale Bars, $100 \mu \mathrm{m}), r f \times 6^{-/-/ n e u r o d: e G F P / i n s u l i n: m C h e r r y ~}$ showed green and red fluoresce in one cell (E), suggesting insulin secreting $\beta$-cell development remained unaffected ( $E$, Scale Bars, $200 \mu \mathrm{m}$ ). $n=10$ fish for the mutant (Mu) and $n=22$ fish for WT (A), $n=23,26$ or 23 fish for the mutant (Mu) and $n=11,12$ or 23 fish for WT (B), $n=8$ fish for the mutant (Mu) and WT (C). $n=5$, Mann-Whitney $U$ test (E). Data are mean \pm S.E.M. A full color version of this figure is available at https://doi. org/10.1530/JME-20-0119. 
the knockout fish specifically regulated the development of non- $\beta$ endocrine cells.

In a further set of experiments, we used neurod: eGFP/insulin:mCherry transgenic fish line to observe the mRNA levels of neurod and insulin in the same cell. When examining neurod:eGFP/insulin:mCherry expression, we found a non-compact islet structure phenotype in the $r f x 6$ knockout fish, which was the same as the H\&E staining results. The islets of $r f x 6$ mutant embryos were loose and disperse in shape and were larger than those in WT embryos. Neurod ${ }^{+}$and ins $^{+}$ cells were both detected in the WT and knockout fish, suggesting that $\beta$-cell development remained unaffected (Fig. 3D and E).

These results show that the $r f x 6$ mutation does not affect the development of endocrine progenitors, but specifically regulate the development of non- $\beta$ endocrine cells.

\section{$R f \times 6$ is not necessary for insulin gene transcription}

To search for genes that might be involved in the $r f x 6$ mutant phenotype, we performed real-time RT-PCR. In $r f \times 6$ knockout fish, pancreatic duodenal homeobox-1 $(p d \times 1)$ and neurod were both upregulated, suggesting that $r f x 6$ acted downstream of these two genes (Fig. 4A). Remarkably, the expression of arx was strongly reduced in $r f x 6$ knockout fish, thus suggesting that $r f x 6$ acted upstream of arx in the regulatory cascade of $\alpha$-cell development (Collombat et al. 2003, Wang et al. 2007, Dorrell et al. 2011). While glucagon, somatostatin and ghrelin were downregulated, insulin was unchanged in rfx6 knockout fish (Dryja et al. 1993, Støy et al. 2007, Edghill et al. 2008) (Fig. 4B, C and D). The expression of key regulators of islet $\beta$-cell development, including mafa, was also normal in the $r f x 6$ knockout fish (Rual et al. 2005, Desai et al. 2008, Papizan et al. 2011, Taylor et al. 2013) (Fig. 4E). As glut2 is also expressed in zebrafish pancreatic $\beta$ cells, we detected the mRNA level of glut2 (Marín-Juez et al. 2015). The results showed that reduced expression in the mutant fish (Fig. 4E). These results were consistent with RNAseq of RNA isolated from the adult pancreas of WT fish and $r f x 6$ knockout fish.

In summary, $r f x 6$ specifically regulates pancreatic islet non- $\beta$-cell development and may decrease insulin synthesis at the translational but not transcriptional level in zebrafish (Fig. 4F).

\section{$R f \times 6$ regulates expressed gene related to protein translation}

To better understand the precise function of $r f x 6$ in the zebrafish, especially the gap between insulin mRNA and protein, high-throughput RNAseq was performed. As it is very hard to dissect pancreas from the adult zebrafish, we isolated pancreas from 5 zebrafish for each group, then mix them for RNAseq. Solexac DNA libraries were constructed from the pancreas of WT and knockout zebrafish. The criteria of threshold $\geq 1.5$ or $\leq 0.67$ in expression were chosen as significantly upregulated or downregulated genes. Using these criteria, a total of 5821 genes were significantly differentially expressed, including 2734 upregulated genes and 3087 downregulated genes.
A
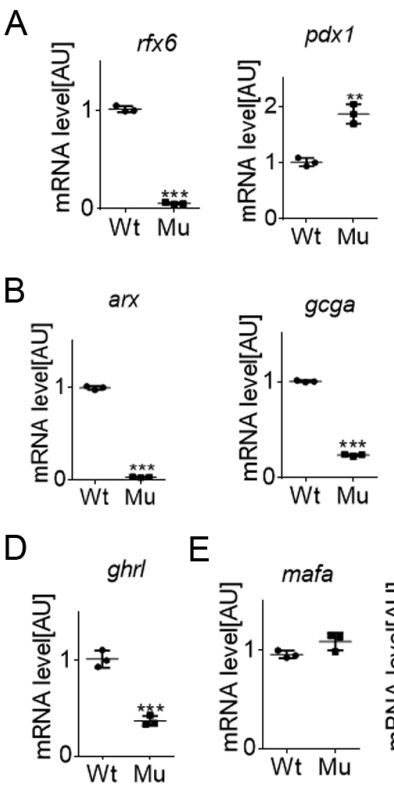

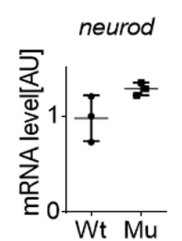

C

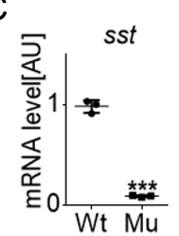

F
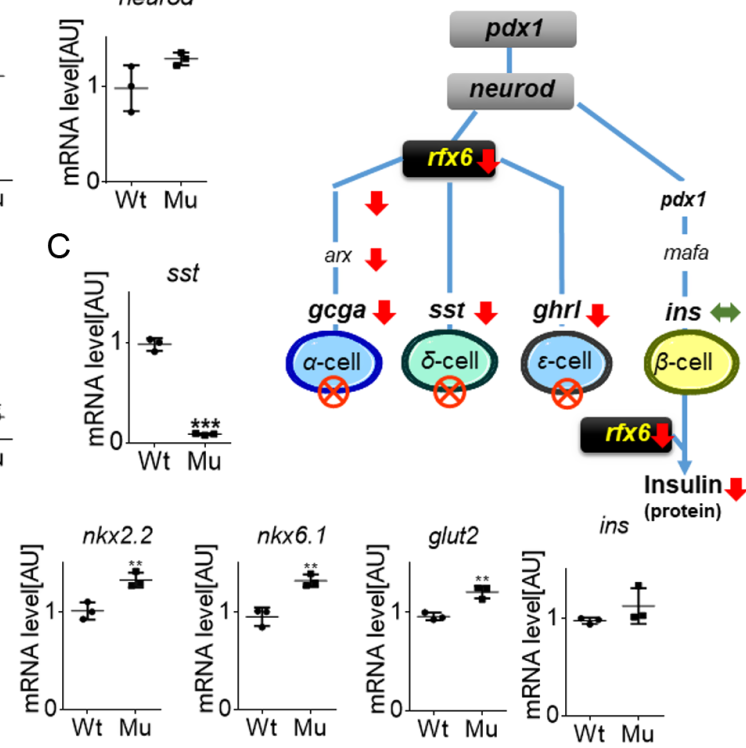

Figure 4

The mRNA levels of pancreas transcription factors. The pancreas of the zebrafish were extracted from the mutant $(\mathrm{Mu})$ and $\mathrm{WT}$ fish at the stage of 3-month. (A, B, C, D and E) The mRNA levels of $p d x 1$ and neurod were upregulated $(A)$ and $r f \times 6$ (A), arx, glucagon (gcga) (B), somatostatin (sst) (C) and ghrelin (ghrl) (D) were downregulated, while the mRNA levels of insulin (ins) remained unchanged in the adult knockout fish (Mu). (F) Summary of endocrine cell development in the rfx 6 knockout fish. $n=6$ (A, B, C, D and E) for the mutant $(\mathrm{Mu})$ and $\mathrm{WT}$, three independent experiments $(\mathrm{A}, \mathrm{B}, \mathrm{C}, \mathrm{D}$ and $\mathrm{E})$. $* P<0.05, * * P<$ $0.01, * * * P<0.001$; Mann-Whitney $U$ test (A, B, C, $D$ and $E)$. Data are mean \pm S.E.M. A full color version of this figure is available at https://doi. org/10.1530/JME-20-0119. 
The Clusters of orthologous groups (COG) enrichment analysis and Kyoto Encyclopedia of Genes and Genomes (KEGG) pathway enrichment analysis were provided by DAVID Bioinformatics Resources 6.7 (Huang et al. 2008) (http://david.abcc.ncifcrf.gov). Among the 5821 differentially expressed genes, DAVID provided functional annotation for 4972 genes (Fig. 5A). KEGG pathway analysis showed that spliceosome pathway was ranked No 1 ( $P=2.40 \mathrm{E}-07)$. Spliceosome pathway was represented by three upregulated and 65 downregulated genes (data not shown). We performed qPCR of hhex and pax6 in the mutant zebrafish. They were no significant change in the mutant fish compared with WT fish. These data meant that hormone- endocrine+ (NeuroD+) cell population was molecularly considered as $\alpha / \delta$ cells, but just not producing hormone. Real-time RT-PCR showed that the expression trends of nine randomly selected genes including snrnp, usp39, eftud, imp3, dhx15, cpsf3, mil1, mcts1 and rbm25 were consistent with RNAseq results. Differentially expressed genes from the mutant zebrafish were mapped to the spliceosome pathways. However, the mRNA sequence of insulin was unchanged in the $r f x 6$ knockout fish, suggesting that the splicing of insulin was normal.

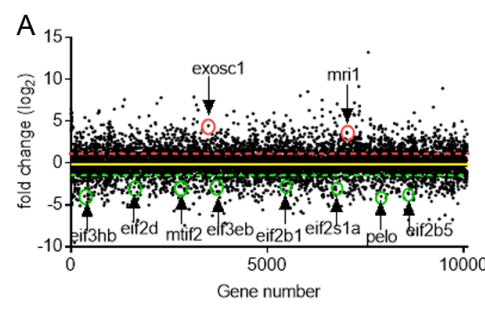

C
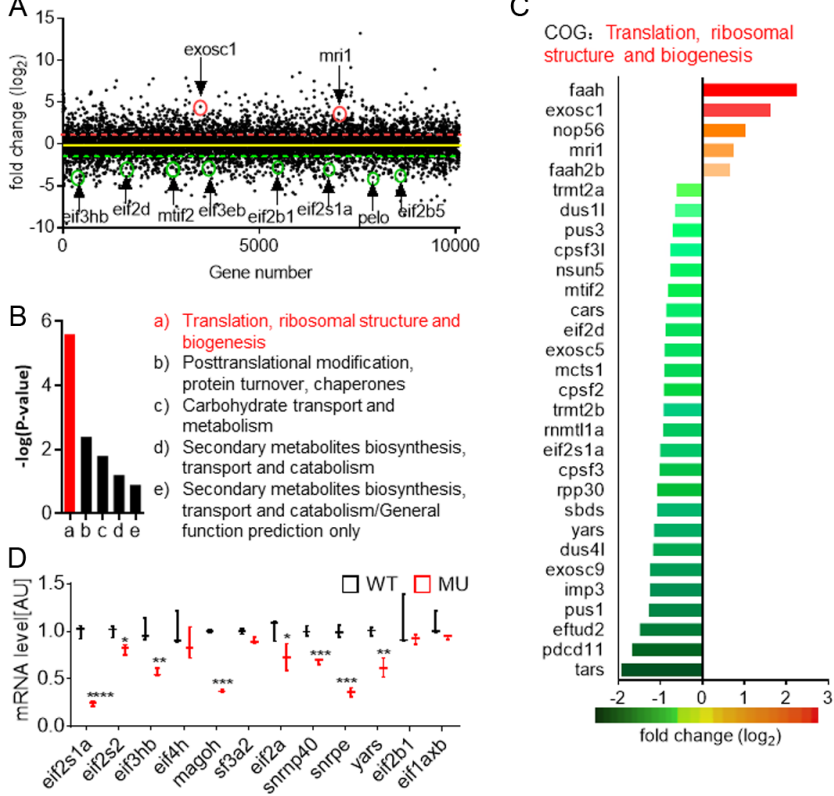

Figure 5

COG ontology enrichment analysis of RNAseq results. (A) RNAseq showed a total of 5821 genes were significantly differentially expressed. (B, C, D and E) COG Ontology enrichment analysis showed that 'Translation, ribosomal structure and biogenesis' may be related with $r f x 6$ gene (B). Differentially expressed genes related with translation were listed (C), which was selectively confirmed by real-time RT-PCR (C). A full color version of this figure is available at https://doi.org/10.1530/JME-20-0119.
COG ontology enrichment analysis showed that the differentially expressed gene may be mainly related to translation, ribosomal structure and biogenesis $(P=2.70 \mathrm{E}-06)$ (Fig. 5B). There was also a statistically significant amount of mapped genes for other functions, such as posttranslational modification. Meanwhile, the detection of real-time RT-PCR showed that the expression trends of 12 randomly selected genes including eif2s1, eif $2 g 2$, eif3hb, eif4h, magoh, sf3a2, eif2a, snrnp40, snrpe, yars, eif2ba and eif1axb, detected by the two methods were consistent. These results suggested that the RNAseq results were reliable (Fig. 5C and D).

\section{Rfx 6 directly binds to promoter and enhancer of eif2ak1 and eif5}

To determine whether translation-related genes might be the direct targets of $r f x 6$, we performed ChIPseq experiments in the pancreas of C57/BL6 mouse. We used C57/BL6 mouse instead of the zebrafish for several reasons. First, zebrafish is too small to get enough pancreas to perform the experiments. Another reason is antibody. We tried but failed to find a good ChIP-grade antibody for zebrafish. The most important is to avoid the confounding factor caused by abnormal development of $r f x 6$ knockout fish.

Here, 4146 unique peaks in the genome region of the adult murine pancreas were captured. Most of the binding peaks were located in intron or intergenic regions. Over 1000 genes were captured as Rfx6-binding genes in the pancreas. One peak was detected in the eif5 promoter region. Similarly, a peak was observed in a conserved region of intron 1 and intron 4 of the eif $2 a k 1$ gene. Eif $2 a k 1$ plays an important role at the translation initiation level to downregulate protein synthesis in response to stress (Lu et al. 2001, Zismanov et al. 2016). Eif5 is a GTPaseactivating protein specific to Eif2, which may act as a part of pre-initiation complex assembly and ensure correct AUG selection during eukaryotic translation initiation (Nanda et al. 2013). Quantitative ChIP experiments using a $r f x 6$ antibody, confirmed that endogenous $r f x 6$ binds to both promoter and enhancer region of eif2ak1 (Fig. $6 \mathrm{~A}$ and $\mathrm{B}) . R f x 6$ can also bind to the promoter region of eif5 (Fig. 6C). These findings were consistent with qRT-PCR results in the zebrafish (Fig. 6D). ChIPseq results also showed that $r f \times 6$ can bind to tars and dus $4 l$, which were closely related with translation. Taken together, our results suggest that $r f x 6$ is a direct positive transcriptional regulator of eif2ak1 and eif5 and that the downregulation of these genes could impact insulin translation which is consistent with RNAseq results. 


\section{Discussion}

The pancreas is an important visceral organ that comprises two portions, the hormone-expressing endocrine islets and the digestive enzyme-expressing exocrine tissues. The endocrine islets are clusters of five types of hormoneproducing cells, $\alpha-, \beta-, \delta$-, PP- and $\varepsilon$-cells, which produce glucagon, insulin, somatostatin, pancreatic polypeptide and ghrelin, respectively. In this study, we showed that $r f x 6$ was necessary for the development of non- $\beta$ endocrine cells in fetal zebrafish. $R f x 6$ is also involved in the formation of a compact islet structure. Postnatal $r f x 6$ knockout fish exhibited a diabetic phenotype resulting from the failure of insulin translation, suggesting that $r f x 6$ was not only a transcription factor but also a translation initiator (Fig. 4F).

Although much of the current understanding of pancreas organogenesis has relied on mouse and human genetic studies, the use of zebrafish has played an important role in studies of genetic pathways especially the early phases of pancreatic development (Moss et al. 2009). Previous studies of zebrafish $r f x 6$ have used morpholinos to focus on its role in the development of pancreas. The predicted zebrafish Rfx6 protein displays $60 \%$ sequence identity with the mouse Rfx6. All mouse Rfx proteins could be identified in zebrafish. However, rfx6 was not present in the gut endoderm, which meant differences in the mechanisms controlling endoderm and/or pancreas specification between the mouse and zebrafish. One study on $r f x 6$ morphants showed that $r f x 6$ was a transcription factor affecting the development of glucagon-, ghrelin- and somatostatin-expressing cells, while insulin-expressing cell remained normal (Soyer et al. 2010). Another study showed that knockdown of RFX6 in xenopus led to a loss of both endocrine and exocrine gene expression, including insulin expression (Pearl et al. 2011). These conflicting results, which may be due to the limitations of morpholinos, leave the function of $r f x 6$ in pancreatic islet cell development unclear. The recently discovered CRISPR/Cas9 system can create insertion and deletion mutations (indels) at specifically targeted sites, thereby causing gene disruption (Soyer et al. 2010, Le et al. 2013, Mali et al. 2013, Qi et al. 2013). This system has been used for gene knockout studies in several model species, including nematodes, fruit flies, zebrafish and mice (Dai et al. 2012, van Schendel et al. 2015, Zhang et al. 2016a,b).

Here, we generated zebrafish $r f x 6$ knockout lines using the CRISPR/Cas9 system. Most homozygous mutants died at approximately 7-14 dpf, with 5\% surviving as infertile adults. In situ hybridization and qPCR results showed that $r f x 6$ is necessary for the development of non- $\beta$ endocrine cells in natal zebrafish. However, $\beta$-cell development and insulin transcription was not affected by the $r f \times 6$ knockout fish. Thus, the results mean that $r f x 6$ is necessary for the development of non- $\beta$ endocrine cells in fetal zebrafish.

The few surviving $r f x 6$ knockout fish exhibited a gaunt body type and high blood glucose levels, both of which are characteristic of diabetes. These phenomena contradicted our qPCR results which showed insulin gene expression were not altered. To explain this controversy,

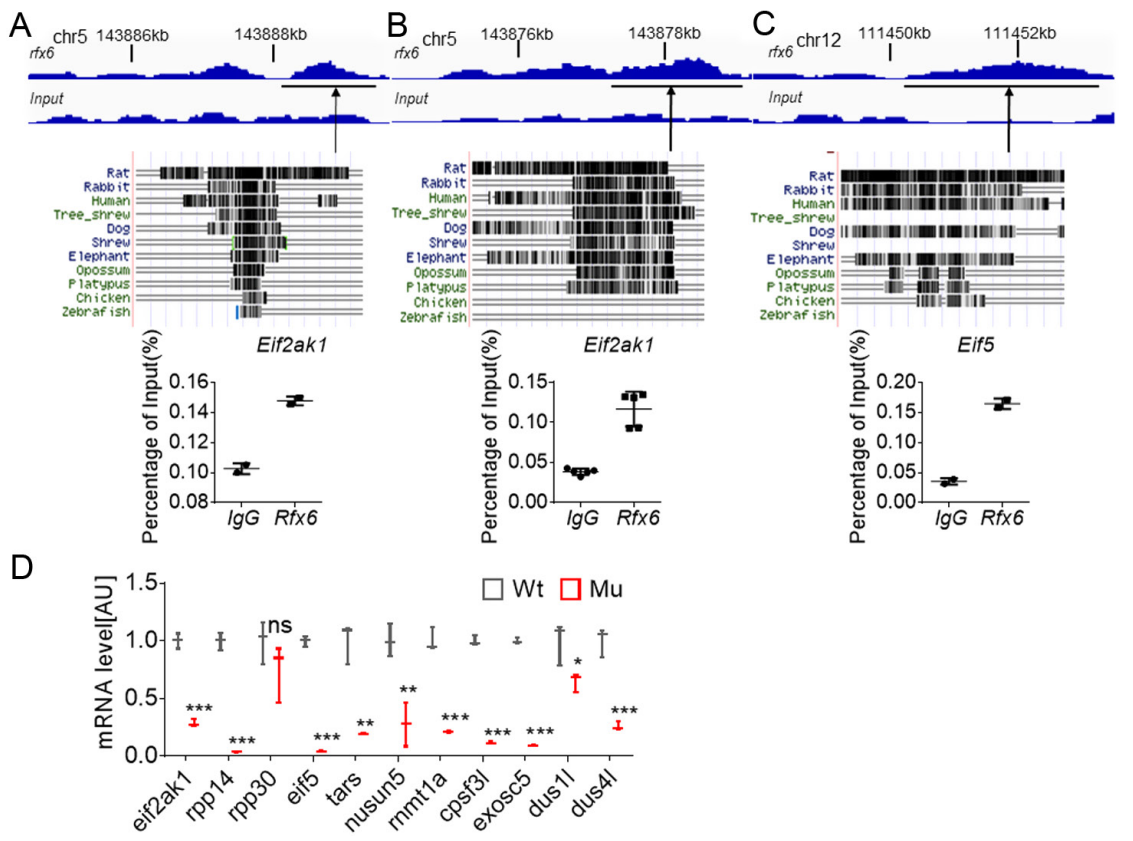

\section{Figure 6}

ChIPseq analysis. (A, B and C) ChIPseq and ChIP qPCR results showed that $R f \times 6$ can directly bind to promoter (A) and enhancer (B) of eif2ak1 and promoter of eif5 gene (C). (D) qRT-PCR results showed that eif2ak1, eif5 and other genes were downregulated in $r f \times 6$ knockout zebrafish. A full color version of this figure is available at https:// doi.org/10.1530/JME-20-0119. 
we further detected the level of insulin protein in postnatal $r f x 6$ knockout fish. Insulin protein was dramatically decreased in the $r f x 6$ knockout fish compared with that of control, thus indicating that insulin transcripts failed to translate to insulin protein. The decreased level of insulin protein may be the cause of diabetic phenotype in postnatal.

We suspected there may be two reasons to explain the gap between normal insulin mRNA level and decreased insulin protein level in postnatal fish. First is the islet structure. The generation of insulin-producing pancreatic $\beta$-cells from human pluripotent stem cells (hPSC) gives the possibility of drug discovery and cell transplantation therapy in diabetes (D'Amour et al. 2005, Cheng et al. 2012, Hrvatin et al. 2014). Stem-cell-derived $\beta$-cells secrete human insulin into the serum of mice only shortly after transplantation. This indicates that not only maturation by upregulation of key $\beta$ cell regulators like MAFA but also the structure of islet may be important for insulin protein secretion. Our study showed that the islets of the rfx6 mutant embryos were loose and disperses in shape and were larger than those in WT embryos. H\&E staining experiments showed that WT islets presented compact and dense shapes, whereas the islets of $r f x 6$ knockout fish were not compact. We did not find ECM or cell adhesion pathway changing in the RNAseq. While some cell adhesion factors including rap1b, gon4l and crb2a were decreased in the RNAseq. This information may explain the structural changes in KO islets. Our model demonstrates that without integrant islets structure, insulin-producing pancreatic $\beta$-cells cannot secret insulin.

Another reason for the gap between normal insulin mRNA level and decreased insulin protein level may be the dysfunction of insulin translation. Previous study showed that transcription factor PAX6 was crucial for $\beta$-cell function through controlling some key genes involved in insulin biosynthesis (Gosmain et al. 2012). RNAseq technology has been successfully applied to several disease models of zebrafish. However, none has used RNAseq approach to elucidate $r f x 6$ precise function in zebrafish. $R f x 6$ is a member of the $R F X$ family of transcription factors, so a better understanding of transcriptome changes in $r f x 6$ mutant may help to analyze the phenotype and find the potential downstream target genes regulated by $r f x 6$. In our study, we performed a highthroughput RNAseq of isolated pancreas from adult $r f x 6$ mutant and control. Interestingly, DAVID enrichment analysis showed that the differentially expressed genes were enriched in 'Translation, ribosomal structure and biogenesis'. It's interesting why and how $r f x 6$ defect may cause the downregulation of a number of translationrelated genes. To avoid the confounding factor caused by abnormal development of $r f x 6$ knockout fish, we performed ChIPseq using normally developed islet $\beta$-cells from C57/BL6 mice. Previous study performed ChIP-seq experiments performed in the mouse $\mathrm{b}$ cell line Min6 transfected with HA-tagged Rfx6 (Piccand et al. 2014). Here, we performed ChIP-seq in the mouse pancreas directly. Our previous results found that $R f x 6$ play an important role in pancreas development in adult mice (Cheng et al. 2019). In this study, we found that $r f x 6$ was a direct positive transcriptional regulator of eif $2 a k 1$ and eif5, which played important roles at the translation initiation level. This can explain why the mRNA level of insulin remained unchanged, while the protein level of insulin dramatically decreased. To our knowledge, this study is the first report that $r f x 6$ is involved in controlling insulin protein translation.

Differentially expressed genes from the knockout fish were mapped to the translation signaling pathways (Walsh \& Mohr 2011) (Fig. 7). Regulated mRNA translation can be subdivided into three stages including initiation, elongation and termination, each of which requires specific factors. At the initiation stage, eif 1 and eif1a, which can bind to 40s ribosome, along with eif2, eif $4 a$, eif4e, eif $4 g$, eif $4 h$, eif5 were downregulated in the $r f x 6$ knockout fish. Importantly, $r f x 6$ can directly bind to the promoter and enhancer elements of eif2ak1 or eif5. $e e f 2 k$, which has an inhibition role in transitioning from initiation to elongation, was upregulated. Termination and re-initiation may be linked through pabp with erf1, pelo, gsgt. The genes were downregulated in the $r f x 6$ knockout fish. Based on the above data, our results suggest a novel function of transcription factor $r f x 6$ in protein translation, which may explain the normal insulin mRNA level but dramatically decreased insulin protein level in postnatal stage.

Our study is limited due to we only focus on insulin protein translation, while protein translation is a global impairment. We will use pdx1-cre mice interlink $R f x 6$ conditional knockout mice in the future to detect the detail function of RFX6.

In our study, we found that $r f x 6$ was necessary for the development of non- $\beta$ endocrine cells in fetal zebrafish, which may be one reason for diabetes. While in postnatal fish, mutation of $r f x 6$ causes dysfunction of insulin translation, which may be another reason for diabetes. In conclusion, mutation of $r f x 6$ disrupts islet non- $\beta$-cell development and insulin translation. A transcription factor regulating the transcription of genes involved in 


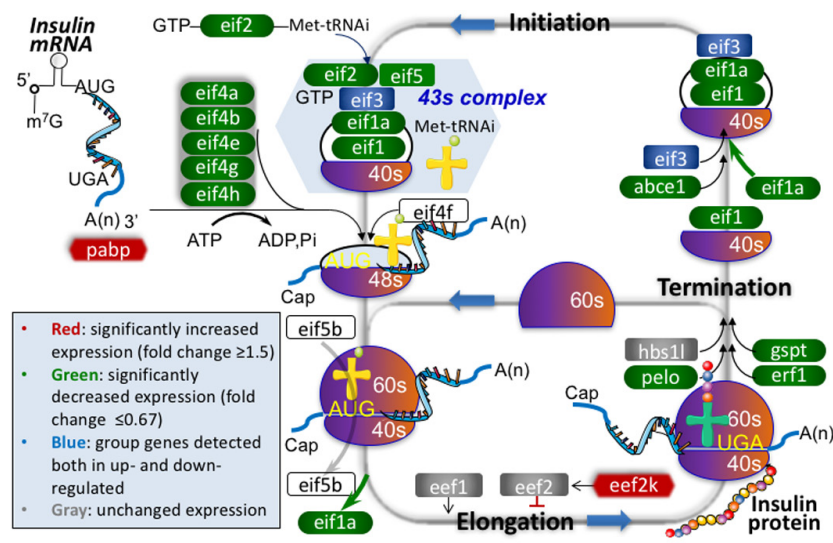

Figure 7

Translation pathway of the $r f \times 6$ knockout fish. The mostly enriched translation pathway for genes differentially expressed (fold-change $\geq 1.5$ or $\leq 0.67$ ) in the $r f x 6$ mutant was shown by DAVID database. Red: significantly increased expression (fold-change $\geq 1.5$ ); Green: significantly decreased expression (fold-change $\leq 0.67$ ); Blue: genes detected both in up and downregulated groups; Gray: unchanged expression. $n=2$ (A, B, C and $\mathrm{E}$ ) for the mutant (Mu) and $\mathrm{WT}, n=6(\mathrm{D}, \mathrm{E})$ for the mutant $(\mathrm{Mu})$ and WT, three independent experiments (D, E). ${ }^{*} P<0.05$, $* * P<0.01$, $* \star * P<$ 0.001; Mann-Whitney $U$ test (D). Data are mean \pm S.E.M. A full color version of this figure is available at https://doi.org/10.1530/JME-20-0119.

mRNA translation may represent a new mechanism and treatment strategy for diabetes.

\section{Declaration of interest}

The authors declare that there is no conflict of interest that could be perceived as prejudicing the impartiality of the research reported.

\section{Funding}

This work was supported by grants from National Key R\&D Program of China (2017YFC0909600) and National Natural Science Foundation of China (81930019) to J K Y, National Natural Science Foundation of China $(81800688,82070890)$ to $\mathrm{J}$ L, and Beijing Municipal Administration of Hospitals Incubating Program (PX2019006) to J L.

\section{Data and resource availability}

The datasets generated during and/or analyzed during the current study are available from the corresponding author upon reasonable request. The $r f \times 6$ mutant zebrafish model generated during and/or analyzed during the current study is available from the corresponding author upon reasonable request.

\section{Author contribution statement}

J KY conceived the idea for the study, designed the experiments and wrote the manuscript. J L, C C, and Q W designed and performed the experiments, and wrote the manuscript. H S performed the experiments. B Z helped with the interpretation of the results and approved the final version of the manuscript.

(c) 2021 Society for Endocrinology Published by Bioscientifica Ltd. Printed in Great Britain

\section{Acknowledgements}

The authors thank the participants and staff of the studies for valuable contributions.

\section{References}

Chandra V, Albagli-Curiel O, Hastoy B, Piccand J, Randriamampita C, Vaillant E, Cavé H, Busiah K, Froguel P, Vaxillaire M, et al. 2014 RFX6 regulates insulin secretion by modulating $\mathrm{Ca} 2+$ homeostasis in human $\beta$ cells. Cell Reports 9 2206-2218. (https://doi.org/10.1016/j. celrep.2014.11.010)

Cheng X, Ying L, Lu L, Galvão AM, Mills JA, Lin HC, Kotton DN, Shen SS, Nostro MC, Choi JK, et al. 2012 Self-renewing endodermal progenitor lines generated from human pluripotent stem cells. Cell Stem Cell 10 371-384. (https://doi.org/10.1016/j.stem.2012.02.024)

Cheng C, Lu J, Cao X, Yang FY, Liu JY, Song LN, Shen H, Liu C, Zhu XR, Zhou JB, et al. 2019 Identification of Rfx6 target genes involved in pancreas development and insulin translation by ChIP-seq. Biochemical and Biophysical Research Communications 508 556-562. (https://doi.org/10.1016/j.bbrc.2018.11.133)

Collombat P, Mansouri A, Hecksher-Sorensen J, Serup P, Krull J, Gradwohl G \& Gruss P 2003 Opposing actions of Arx and Pax4 in endocrine pancreas development. Genes and Development 17 25912603. (https://doi.org/10.1101/gad.269003)

Concepcion JP, Reh CS, Daniels M, Liu X, Paz VP, Ye H, Highland HM, Hanis CL \& Greeley SA 2014 Neonatal diabetes, gallbladder agenesis, duodenal atresia, and intestinal malrotation caused by a novel homozygous mutation in RFX6. Pediatric Diabetes 15 67-72. (https:// doi.org/10.1111/pedi.12063)

Dai C, Brissova M, Hang Y, Thompson C, Poffenberger G, Shostak A, Chen Z, Stein R \& Powers AC 2012 Islet-enriched gene expression and glucose-induced insulin secretion in human and mouse islets. Diabetologia 55 707-718. (https://doi.org/10.1007/s00125-011-2369-0)

D'Amour KA, Agulnick AD, Eliazer S, Kelly OG, Kroon E \& Baetge EE 2005 Efficient differentiation of human embryonic stem cells to definitive endoderm. Nature Biotechnology 23 1534-1541.

Desai S, Loomis Z, Pughbernard A, Schrunk J, Doyle MJ, Minic A, Mccoy E \& Sussel L 2008 Nkx2.2 regulates cell fate choice in the enteroendocrine cell lineages of the intestine. Developmental Biology 313 58-66. (https://doi.org/10.1016/j.ydbio.2007.09.047)

Dorrell C, Schug J, Lin CF, Canaday PS, Fox AJ, Smirnova O, Bonnah R, Streeter Jr PR, Stoeckert CJ, Kaestner KH, et al. 2011 Transcriptomes of the major human pancreatic cell types. Diabetologia $5 \mathbf{4}$ 2832-2844. (https://doi.org/10.1007/s00125-011-2283-5)

Dryja TP, Berson EL, Rao VR \& Oprian DD 1993 Heterozygous missense mutation in the rhodopsin gene as a cause of congenital stationary night blindness. Nature Genetics 4 280-283. (https://doi.org/10.1038/ ng0793-280)

Edghill EL, Flanagan SE, Patch AM, Boustred C, Parrish A, Shields B, Shepherd MH, Hussain K, Kapoor RR, Malecki M, et al. 2008 Insulin mutation screening in 1044 patients with diabetes: mutations in the INS gene are a common cause of neonatal diabetes but a rare cause of diabetes diagnosed in childhood or adulthood. Diabetes 57 10341042. (https://doi.org/10.2337/db07-1405)

Gosmain Y, Katz LS, Masson MH, Cheyssac C, Poisson C \& Philippe J 2012 Pax6 is crucial for $\beta$-cell function, insulin biosynthesis, and glucose-induced insulin secretion. Molecular Endocrinology 26 696709. (https://doi.org/10.1210/me.2011-1256)

Hrvatin S, O'Donnell CW, Deng F, Millman JR, Pagliuca FW, Diiorio P, Rezania A, Gifford DK \& Melton DA 2014 Differentiated human stem cells resemble fetal, not adult, $\beta$ cells. PNAS $1113038-3043$. (https://doi.org/10.1073/pnas.1400709111)

Huang DW, Sherman BT \& Lempicki RA 2008 Systematic and integrative analysis of large gene lists using DAVID bioinformatics resources. Nature Protocols 4 44-57. 
Intine RV, Olsen AS \& Sarras Jr MP 2013 A zebrafish model of diabetes mellitus and metabolic memory. Journal of Visualized Experiments $\mathbf{7 2}$ e50232. (https://doi.org/10.3791/50232)

Le C, Ran FA, Cox D, Lin S, Barretto R, Habib N, Hsu PD, Wu X, Jiang W \& Marraffini LA 2013 Multiplex genome engineering using CRISPR/Cas systems. Trends in Genetics 32815.

Lu L, Han AP \& Chen JJ 2001 Translation initiation control by hemeregulated eukaryotic initiation factor $2 \alpha$ kinase in erythroid cells under cytoplasmic stresses. Molecular and Cellular Biology 21 79717980. (https://doi.org/10.1128/MCB.21.23.7971-7980.2001)

Mali P, Yang L, Esvelt KM, Aach J, Guell M, Dicarlo JE, Norville JE \& Church GM 2013 RNA-guided human genome engineering via Cas9. Science 339 823-826. (https://doi.org/10.1126/science.1232033)

Marín-Juez R, Rovira M, Crespo D, van der Vaart M, Spaink HP \& Planas JV 2015 GLUT2-mediated glucose uptake and availability are required for embryonic brain development in zebrafish. Journal of Cerebral Blood Flow and Metabolism 35 74-85. (https://doi. org/10.1038/jcbfm.2014.171)

Moss JB, Koustubhan P, Greenman M, Parsons MJ, Walter I \& Moss LG 2009 Regeneration of the pancreas in adult zebrafish. Diabetes $\mathbf{5 8}$ 1844-1851. (https://doi.org/10.2337/db08-0628)

Namba T 2019 BAP31 regulates mitochondrial function via interaction with Tom40 within ER-mitochondria contact sites. Science Advances 5 eaaw1386. (https://doi.org/10.1126/sciadv.aaw1386)

Nanda JS, Saini AK, Muñoz AM, Hinnebusch AG \& Lorsch JR 2013 Coordinated movements of eukaryotic translation initiation factors eIF1, eIF1A, and eIF5 trigger phosphate release from eIF2 in response to start codon recognition by the ribosomal preinitiation complex. Journal of Biological Chemistry 288 5316-5329. (https://doi. org/10.1074/jbc.M112.440693)

Obholzer N, Wolfson S, Trapani JG, Mo W, Nechiporuk A, BuschNentwich E, Seiler C, Sidi S, Söllner C, Duncan RN, et al. 2008 Vesicular glutamate transporter 3 is required for synaptic transmission in zebrafish hair cells. Journal of Neuroscience 282110 2118. (https://doi.org/10.1523/JNEUROSCI.5230-07.2008)

Papizan JB, Singer RA, Tschen SI, Dhawan S, Friel JM, Hipkens SB, Magnuson MA, Bhushan A \& Sussel L 2011 Nkx2.2 repressor complex regulates islet $\beta$-cell specification and prevents $\beta$-to- $\alpha$-cell reprogramming. Genes and Development 25 2291-2305. (https://doi. org/10.1101/gad.173039.111)

Patel KA, Kettunen J, Laakso M, Stančáková A, Laver TW, Colclough K, Johnson MB, Abramowicz M, Groop L, Miettinen PJ, et al. 2017 Heterozygous RFX6 protein truncating variants are associated with MODY with reduced penetrance. Nature Communications 8 888. (https://doi.org/10.1038/s41467017-00895-9)

Pauerstein PT, Sugiyama T, Stanley SE, McLean GW, Wang J, Martín MG \& Kim SK 2015 Dissecting human gene functions regulating islet development with targeted gene transduction. Diabetes 64 3037-3049. (https://doi.org/10.2337/db15-0042)

Pearl EJ, Jarikji Z \& Horb ME 2011 Functional analysis of Rfx6 and mutant variants associated with neonatal diabetes. Developmental Biology 351 135-145. (https://doi.org/10.1016/j.ydbio. 2010.12.043)

Piccand J, Strasser P, Hodson DJ, Meunier A, Ye T, Keime C, Birling MC, Rutter GA \& Gradwohl G 2014 Rfx6 maintains the functional identity of adult pancreatic $\beta$ cells. Cell Reports 9 2219-2232. (https://doi.org/10.1016/j.celrep.2014.11.033)

Pisharath H, Rhee JM, Swanson MA, Leach SD \& Parsons MJ 2007 Targeted ablation of beta cells in the embryonic zebrafish pancreas using E. coli nitroreductase. Mechanisms of Development 124 218-229. (https://doi.org/10.1016/j.mod.2006.11.005)
Qi LS, Larson MH, Gilbert LA, Doudna JA, Weissman JS, Arkin AP \& Lim WA 2013 Repurposing CRISPR as an RNA-guided platform for sequence-specific control of gene expression. Cell 152 1173-1183. (https://doi.org/10.1016/j.cell.2013.02.022)

Rual JF, Venkatesan K, Hao T, Hirozane-Kishikawa T, Dricot A, Li N, Berriz GF, Gibbons FD, Dreze M, Ayivi-Guedehoussou N, et al. 2005 Towards a proteome-scale map of the human protein $i$ protein interaction network. Nature 437 1173-1178. (https://doi. org/10.1038/nature04209)

Smith SB, Qu HQ, Taleb N, Kishimoto NY, Scheel DW, Lu Y, Patch AM, Grabs R, Wang J, Lynn FC, et al. 2010 Rfx6 directs islet formation and insulin production in mice and humans. Nature 463 775-780. (https://doi.org/10.1038/nature08748)

Soyer J, Flasse L, Raffelsberger W, Beucher A, Orvain C, Peers B, Ravassard P, Vermot J, Voz ML, Mellitzer G, et al. 2010 Rfx6 is an Ngn3-dependent winged helix transcription factor required for pancreatic islet cell development. Development 137 203-212. (https:// doi.org/10.1242/dev.041673)

Støy J, Edghill EL, Flanagan SE, Ye H, Paz VP, Pluzhnikov A, Below JE, Hayes MG, Cox NJ, Lipkind GM, et al. 2007 Insulin gene mutations as a cause of permanent neonatal diabetes. PNAS $\mathbf{1 0 4} 15040-15044$ (https://doi.org/10.1073/pnas.0707291104)

Taylor BL, Liu FF \& Sander M 2013 Nkx6.1 is essential for maintaining the functional state of pancreatic beta cells. Cell Reports $\mathbf{4}$ 1262-1275. (https://doi.org/10.1016/j.celrep.2013.08.010)

Tong X, Zu Y, Li Z, Li W, Ying L, Yang J, Wang X, He S, Liu D, Zhu Z, et al. 2014 Kctd10 regulates heart morphogenesis by repressing the transcriptional activity of Tbx5a in zebrafish. Nature Communications 5 3153. (https://doi.org/10.1038/ncomms4153)

Toselli CM, Wilkinson BM, Paterson J \& Kieffer TJ 2019 Vegfa/vegfr2 signaling is necessary for zebrafish islet vessel development, but is dispensable for beta-cell and alpha-cell formation. Scientific Reports 9 3594. (https://doi.org/10.1038/s41598-019-40136-1)

van Schendel R, Roerink SF, Portegijs V, van den Heuvel S \& Tijsterman M 2015 Polymerase $\Theta$ is a key driver of genome evolution and of CRISPR/Cas9-mediated mutagenesis. Nature Communications 6 7394. (https://doi.org/10.1038/ncomms8394)

Walsh D \& Mohr I 2011 Viral subversion of the host protein synthesis machinery. Nature Reviews: Microbiology 9 860-875. (https://doi. org/10.1038/nrmicro2655)

Wang H, Brun T, Kataoka K, Sharma AJ \& Wollheim CB 2007 MAFA controls genes implicated in insulin biosynthesis and secretion. Diabetologia 50 348-358. (https://doi.org/10.1007/s00125006-0490-2)

Zhang R, Miner JJ, Gorman MJ, Rausch K, Ramage H, White JP, Zuiani A, Zhang P, Fernandez E, Zhang Q, et al. 2016a A CRISPR screen defines a signal peptide processing pathway required by flaviviruses. Nature 535 164-168. (https://doi.org/10.1038/ nature18625)

Zhang Y, Huang H, Zhang B \& Lin S 2016b Chapter 5 - TALEN- and CRISPR-enhanced DNA homologous recombination for gene editing in zebrafish. Methods in Cell Biology 135 107-120. (https://doi. org/10.1016/bs.mcb.2016.03.005)

Zhu Z, Li QV, Lee K, Rosen BP, González F, Soh CL \& Huangfu D 2016 Genome editing of lineage determinants in human pluripotent stem cells reveals mechanisms of pancreatic development and diabetes. Cell Stem Cell 18 755-768. (https://doi.org/10.1016/j.stem.2016.03.015)

Zismanov V, Chichkov V, Colangelo V, Jamet S, Wang S, Syme A, Koromilas AE \& Crist C 2016 Phosphorylation of eIF2 $\alpha$ is a translational control mechanism regulating muscle stem cell quiescence and self-renewal. Cell Stem Cell 18 79-90. (https://doi. org/10.1016/j.stem.2015.09.020)

Received in final form 29 November 2020

Accepted 16 December 2020

Accepted Manuscript published online 23 December 2020
(C) 2021 Society for Endocrinology Published by Bioscientifica Ltd. Printed in Great Britain 